\title{
ON FOURIER TRANSFORMS. III $\dagger$
}

BY
A. C. OFFORD

1. Introduction. In this paper we are concerned with functions connected by the relations

$$
\begin{aligned}
& F(x)=(2 \pi)^{-1 / 2} \int_{-\infty}^{\infty} f(u) e^{-i x u} d u, \\
& f(x)=(2 \pi)^{-1 / 2} \int_{-\infty}^{\infty} F(u) e^{i x u} d u .
\end{aligned}
$$

The integrals will usually be interpreted in the sense of Cesàro, i.e., the integral $\int_{-\infty}^{\infty} \phi(u) d u$ is said to be summable $(C, k), k>0$, if

$$
\int_{-\omega}^{\omega}\left(1-\frac{|u|}{\omega}\right)^{k} \phi(u) d u
$$

tends to a limit as $\omega$ tends to infinity.

Definition 1. Write

$$
F(x, \omega)=(2 \pi)^{-1 / 2} \int_{-\omega}^{\omega}\left(1-\frac{|u|}{\omega}\right) f(u) e^{-i x u} d u .
$$

Suppose that $F(x, \omega)$ is in $L^{p}(-\infty, \infty), \S 1 \leqq p \leqq \infty$, for all $\omega$ and that there is a function $F(x)$ such that, if $1 \leqq p<\infty$,

$$
\lim _{\omega \rightarrow \infty} \int_{-\infty}^{\infty}|F(x)-F(x, \omega)|^{p} d x=0,
$$

or in case $\dot{p}=\infty$,

$$
\lim _{\omega \rightarrow \infty} \text { essential upper bound }|F(x)-F(x, \omega)|=0 .
$$

Then we say that $F(x)$ is the Fourier transform in $L^{p}$ of $f(x)$.

This definition is shown to be consistent with the usual one\|l except in the cases $p=1, \infty$.

$\dagger$ Presented to the Society, February 23, 1935; received by the editors April 2, 1934.

$\ddagger$ Keddey Fletcher-Warr student of the University of London.

§ A measurable function $\phi(x)$ is in $L^{p}(a, b), 1 \leqq p<\infty$, if $\int_{a}^{b}|\phi(x)|^{p} d x$ is finite. It is in $L^{\infty}(a, b)$ if it is equivalent to a function which is bounded in $(a, b)$.

|| Cf. Wiener, 12, p. 67; Berry, 2, p. 227. (See Bibliography, on p. 266, for references.) 
Definition 2. A measurable function $f(x)$ is said to belong to the class $H^{p}$, $1<p<\infty$, when it is integrable $L$ in every finite range and such that

$$
\int_{-\infty}^{\infty}|F(x, \omega)| p d x \leqq M^{p},
$$

where $F(x, \omega)$ is given by (1.2) and $M \dagger$ is a number independent of $\omega$. The function $f(x)$ belongs to the class $H^{1}$ if it satisfies (1.3) for $p=1$ and in addition

$$
\int_{e}|F(x, \omega)| d x \leqq \epsilon
$$

whenever the measure of the set $e$ is less than some positive number $\delta(\epsilon)$. When $p=\infty$ the condition (1.3) is to be interpreted as

$$
|F(x, \omega)| \leqq M,
$$

for all $x$ and $\omega$.

Definition 3. A measurable function $F(x)$ belongs to the class $L_{p}^{*}$ if it is in $L^{p}(-\infty, \infty), 1 \leqq p<\infty$, and such that

$$
(2 \pi)^{-1 / 2} \int_{-\infty}^{\infty} \frac{e^{i x u}-1}{i u} F(u) d u
$$

converges to an indefinite Lebesgue integral. The class $L_{\infty}^{*}$ is the class of all the bounded functions $F(x)$ for which the expression

$$
\begin{aligned}
(2 \pi)^{-1 / 2}\left\{\int_{-1}^{1} \frac{e^{i x u}-1}{i u} F(u) d u\right. & \\
& \left.+\int_{1}^{\infty} \frac{e^{i x u}}{i u} F(u) d u+\int_{-\infty}^{-1} \frac{e^{i x u}}{i u} F(u) d u\right\}
\end{aligned}
$$

is summable $(C, 1)$ to an indefinite Lebesgue integral.

Definition 4. A measurable function belongs to the class $H^{p} L^{q}, 1 \leqq p \leqq \infty$, $1 \leqq q \leqq \infty$, if it belongs to both $H^{p}$ and $L^{q}$.

The results of this paper can now be summarized as follows:

(i) If $f(x)$ belongs to $H^{p}, 1 \leqq p \leqq \infty$, then it has a Fourier transform $F(x)$ in $L^{p}$ and the inverse formula (1.12) holds (Theorems 1 and 2).

(ii) If $F(x)$ belongs to $L_{p}^{*}, 1 \leqq p \leqq \infty$, then it is the Fourier transform in $L^{p}$ of a function $f(x)$ which belongs to $H^{p}$ (Theorem 5 ).

$\dagger$ Throughout this paper we shall use $M$ to denote a number not necessarily the same at each occurrence but always independent of the variables under consideration. 
(iii) If $f(x)$ belongs to $H^{p} L^{q}$, then it has a Fourier transform $F(x)$ in $L^{p}$. $F(x)$ belongs to $H^{q}$ and $f(-x)$ is the Fourier transform in $L^{q}$ of $F(x)$ (Theorem 10).

(iv) Thereis complete reciprocity in the class $B^{p} L^{p}, 1 \leqq p \leqq \infty$ (Theorem 12).

It is not assumed in (i) that $f(x)$ belongs to a Lebesgue class and this need not be the case. Hence although the inverse formula (1.12) holds it does not follow that $f(-x)$ is the Fourier transform of $F(x)$ in the sense of Definition 1. The result (ii) is the converse of (i). It is also shown in Theorem 6 that if $f(x)$ belongs to $H^{p}$ then its Fourier transform $F(x)$ belongs to $L_{p}^{*}$.

The results of (iii) and (iv) complete those of (i). A result of particular interest in the Plancherel-Titchmarsh theory of Fourier transforms is the reciprocity in the class $L^{2}$. In (iv) we assert the existence of other classes of functions which also possess this reciprocity. When $1 \leqq p \leqq 2$ the class $H^{p} L^{p}$ is contained in $L^{2}$ but for $p>2$ this need not be the case.

There is an interesting connection between the case $p=\infty$ and functions which are bounded and harmonic in a half plane. We have already discussed this case from this point of view. $\dagger$ However, the arguments there employed are hardly suited for the case $1 \leqq p<\infty$. Accordingly, in this paper we employ an entirely different method using the notion of weak convergence where before we used Fatou's theorem. We shall, however, refer to our previous paper for the proofs of some of the theorems concerning the case $p=\infty$ when they differ from the case $1 \leqq p<\infty$.

One further point requires explanation. It is the substitution of Definition 1 for the definition of the Fourier transform ordinarily given. There are two reasons for this. First, with the ordinary definition, some of the results of this paper, noticeably Theorem 5 , would not be true in the case $p=1$ as has been shown by Hille and Tamarkin. $\ddagger$ Secondly, with Definition 1, not only does $F(x, \omega)$ converge in mean to $F(x)$ but it also converges in the ordinary sense almost everywhere. This enables us to avoid using convergence in mean which is in some respects an advantage. In fact some of the functions we employ do not belong to Lebesgue classes and so the notion of convergence in mean is not always applicable. However, it can be shown by the argument used by Hille and Tamarkin§ that the two definitions are equivalent except when $p=1, \infty$. This we show in Theorem 7 .

2. The class $H^{p}$. In this section we shall prove some of the fundamental results of the paper. In each case we shall have recourse to a series of lemmas.

$\dagger$ Offord, 7. The classes $B^{\infty}$ and $L_{\infty}^{*}$ were described in this paper as the classes $B$ and $B^{*}$ respectively. Some of these results have been extended to Hankel transforms. See 8, and 9.

$\ddagger$ Hille and Tamarkin, 4, p. 773.

§ Loc. cit. 
ThEOREM 1. If $f(x)$ belongs to $H^{p}, 1 \leqq p<\infty$, then the integral

$$
(2 \pi)^{-1 / 2} \int_{-\infty}^{\infty} f(u) e^{-i x u} d u
$$

is summable $(C, 1)$ almost everywhere, to a function $F(x)$ which belongs to the class $L^{p}(-\infty, \infty)$. The function $F(x)$ is the Fourier transform in $L^{p}$ of $f(x)$ and

$$
f(x)=(2 \pi)^{-1 / 2} \int_{-\infty}^{\infty} F(u) e^{i x u} d u
$$

almost everywhere.

THEOREM 2. If $f(x)$ belongs to $H^{\infty}$, then the integral (2.1) is boundedly summable $(C, 1)$ almost everywhere to a bounded function $F(x)$ which is the Fourier transform in $L^{\infty}$ of $f(x)$ and

$$
f(x)=(2 \pi)^{-1 / 2} \int_{-\infty}^{\infty} F(u) e^{i x u} d u
$$

almost everywhere.

Theorem 1 follows from Lemmas 3 and 4 . The proof of Theorem 2 is given elsewhere. $†$ Possibly the most important result in this connection is Parseval's theorem which takes the following forms according to the value of $p$.

Theorem 3. Let $f(x)$ belong to $H^{p}, 2 \leqq p \leqq \infty$. Let $G(x)$ belong to $L^{p^{\prime}}$ $(-\infty, \infty)$, where $p^{\prime}=p /(p-1)$. Let $g(-x)$ be the Fourier transform $\ddagger$ in $L^{p}$ of $G(x)$ and let $g(x)$ be bounded in every finite range. Then

$$
\int_{-\infty}^{\infty} F(x) G(x) d x=\int_{-\infty}^{\infty} f(x) g(-x) d x
$$

where the first integral is convergent and the second summable $(C, 1)$.

Theorem 4 . Let $f(x)$ belong to $H^{p}, 1 \leqq p \leqq 2$. Let $g(x)$ belong to $L^{p}(-\infty, \infty)$ and let $g(x)$ be bounded in every finite range. Let $G(x)$ be the Fourier transform $\ddagger$ in $L^{p^{\prime}}$ of $g(x)$. Then

$$
\int_{-\infty}^{\infty} F(x) G(x) d x=\int_{-\infty}^{\infty} f(x) g(-x) d x
$$

where the first integral is convergent and the second summable $(C, 1)$.

$\dagger$ Offord, 7, Theorem 1. This theorem only asserts that $(2.2)$ is summable $(C, 2)$ almost everywhere. The complete result is given in Offord, 10 .

$\ddagger$ In the ordinary sense. It is, of course, a classical result that, if $G(x)$ belongs to $L^{p^{\prime}}, 1 \leqq p^{\prime} \leqq 2$, then $G(x)$ has a Fourier transform in $L^{p}$. A similar remark applies also to Theorem 4. 
The proofs of Theorems 3 and 4 are given after Lemma 3. We employ these theorems in establishing the inverse relations in Theorems 1 and 2.

LeMma 1. Let $f(u)$ belong to $H^{p}, 1 \leqq p \leqq \infty$, and let

$$
F(x, \nu)=(2 \pi)^{-1 / 2} \int_{-\nu}^{\nu}\left(1-\frac{|u|}{\nu}\right) f(u) e^{-i x u} d u .
$$

Then there exists a sequence $\left\{\nu_{j}\right\}$ such that $F\left(x, \nu_{j}\right)$ converges weakly, with exponent $p$, to some function $F(x)$ of the class $L^{p}$.

By the definition of the class $H^{p}$ the functions $F(x, \nu)$ are uniformly bounded in $L^{p}$. The lemma is thus a classical result $\dagger$ of the theory of weak convergence.

In the following lemma we consider a function $G(x)$ which is bounded and integrable $L$ in $(-\infty, \infty)$. This function belongs to every Lebesgue class and so has a Fourier transform $g(-u)$ in the classical sense.

Lemma 2. Let $f(x)$ belong to $H^{p}, 1 \leqq p \leqq \infty$, and let a sequence $\left\{\nu_{j}\right\}$ and a function $F(x)$ be defined as in Lemma 1 . Let $G(x)$ be integrable $L$ and bounded in $(-\infty, \infty)$ and let $g(-x)$ be the Fourier transform of $G(x)$. Then

$$
\int_{-\infty}^{\infty} F(u) G(u) d u=\lim _{j \rightarrow \infty} \int_{-v_{j}}^{\nu_{j}}\left(1-\frac{|u|}{\nu_{j}}\right) f(u) g(-u) d u .
$$

Since $G(x)$ belongs to $L(-\infty, \infty)$,

$$
\begin{aligned}
& \int_{-\nu_{j}}^{\nu_{j}}\left(1-\frac{|u|}{\nu_{j}}\right) f(u) g(-u) d u \\
& \quad=(2 \pi)^{-1 / 2} \int_{-\nu_{j}}^{\nu_{j}}\left(1-\frac{|u|}{\nu_{j}}\right) f(u) d u \int_{-\infty}^{\infty} G(x) e^{-i x u} d x \\
& \quad=(2 \pi)^{-1 / 2} \int_{-\infty}^{\infty} G(x) d x \int_{-\nu_{j}}^{\nu_{j}}\left(1-\frac{|u|}{\nu_{j}}\right) f(u) e^{-i x u} d u=\int_{-\infty}^{\infty} F\left(x, \nu_{j}\right) G(x) d x .
\end{aligned}
$$

Now $G(x)$ is bounded and belongs to $L(-\infty, \infty)$. Hence it belongs to $L^{p^{\prime}}$ $(-\infty, \infty), p^{\prime}=p /(p-1)$. Therefore, by Lemma 1 and the general convergence theorem of Hobson and Lebesgue, $\ddagger$

$$
\int_{-\infty}^{\infty} F(x) G(x) d x=\lim _{\nu_{j} \rightarrow \infty} \int_{-v_{j}}^{v_{j}}\left(1-\frac{|u|}{\nu_{j}}\right) f(u) g(-u) d u .
$$

The reader will observe that, in the proof of the case $p=1$, it is at this stage that we require (1.31).

$\dagger$ Cf. Hobson, 5, p. 253, and Banach, 1.

$\ddagger$ Hobson, 5, p. 422, and Lebesgue, 6, p. 52. 
LEMMA 3. If $f(x)$ belongs to $H^{p}, 1 \leqq p \leqq \infty$, then the integral

$$
(2 \pi)^{-1 / 2} \int_{-\infty}^{\infty} f(u) e^{-i x u} d u
$$

is summable $(C, 1)$ almost everywhere to a function $F(x)$ which is the Fourier transform in $L^{p}$ of $f(x)$.

In Lemma 2 take

Then

$$
g(u)=\left\{\begin{array}{cr}
(2 \pi)^{-1 / 2}\left(1-\frac{|u|}{\omega}\right) e^{i x u}, & -\omega \leqq u \leqq \omega, \\
0, & u \leqq-\omega, \omega \leqq u .
\end{array}\right.
$$

$$
G(y)=(2 \pi)^{-1 / 2} \int_{-\infty}^{\infty} g(u) e^{-i y u} d u=\frac{2}{\pi \omega} \frac{\sin ^{2} \frac{1}{2} \omega(x-y)}{(x-y)^{2}} .
$$

The function $G(y)$ obviously satisfies the hypotheses of Lemma 2. Hence, if $F(y)$ is defined as in Lemma 1 , we have

$$
(2 \pi)^{-1 / 2} \int_{-\omega}^{\omega}\left(1-\frac{|u|}{\omega}\right) f(u) e^{-i x u} d u=\frac{2}{\pi \omega} \int_{-\infty}^{\infty} \frac{\sin ^{2} \frac{1}{2} \omega(x-y)}{(x-y)^{2}} F(y) d y .
$$

Now this is Fejér's integral and it is well known that for $1 \leqq p \leqq \infty$ the integral tends to $F(x)$ almost everywhere as $\omega$ tends to infinity. Hence

$$
F(x)=(2 \pi)^{-1 / 2} \lim _{\omega \rightarrow \infty} \int_{-\omega}^{\omega}\left(1-\frac{|u|}{\omega}\right) f(u) e^{-i x u} d u
$$

almost everywhere, the convergence being in $L^{p}$. This is the desired result.

We can now complete the proofs of Theorems 3 and 4.

Proof of Theorem 3. By hypothesis, $f(x)$ belongs to $H^{p}$ and $G(x)$ to $L^{p^{\prime}}$. Hence, by Lemma 3,

$$
\begin{aligned}
\int_{-\infty}^{\infty} & G(x) F(x) d x=(2 \pi)^{-1 / 2} \lim _{\omega \rightarrow \infty} \int_{-\infty}^{\infty} G(x) d x \int_{-\omega}^{\omega}\left(1-\frac{|u|}{\omega}\right) f(u) e^{-i x u} d u \\
& =(2 \pi)^{-1 / 2} \lim _{\omega \rightarrow \infty} \lim _{x \rightarrow \infty} \int_{-x}^{x}\left(1-\frac{|x|}{X}\right) G(x) d x \int_{-\omega}^{\omega}\left(1-\frac{|u|}{\omega}\right) f(u) e^{-i x u} d u \\
& =\lim _{\omega \rightarrow \infty} I,
\end{aligned}
$$

in virtue of the consistence theorem for Cesàro summation. We use $I$ as an abbreviation.

Now $G(x)$ belongs to $L^{p^{\prime}}, 1 \leqq p^{\prime} \leqq 2$. Hence $G(x)$ has a Fourier transform $g(-u)$ in $L^{p}$. Also

$†$ This follows from a classical argument due to Plancherel. See Hobson, 5, pp. 748-49. The proof for bounded convergence is of course the same as the proof for uniform convergence. 


$$
g(-u)=\lim _{X \rightarrow \infty}(2 \pi)^{-1 / 2} \int_{-X}^{X}\left(1-\frac{|x|}{X}\right) G(x) e^{-i x u} d x .
$$

Further the convergence is bounded in every interval in which $g(-u)$ is bounded and so, by hypothesis, in every finite range $|u| \leqq \omega$. Therefore

$$
\begin{aligned}
I & =\lim _{X \rightarrow \infty}(2 \pi)^{-1 / 2} \int_{-\omega}^{\omega}\left(1-\frac{|u|}{\omega}\right) f(u) d u \int_{-X}^{X}\left(1-\frac{|x|}{X}\right) G(x) e^{-i x u} d x \\
& =\int_{-\omega}^{\omega}\left(1-\frac{|u|}{\omega}\right) f(u) g(-u) d u .
\end{aligned}
$$

Hence

$$
\int_{-\infty}^{\infty} G(x) F(x) d x=\lim _{\omega \rightarrow \infty} \int_{-\omega}^{\omega}\left(1-\frac{|u|}{\omega}\right) f(u) g(-u) d u
$$

and this is the desired result.

Proof of Theorem 4. The proof of this theorem is very similar to that of Theorem 3. Here $g(u)$ belongs to $L^{p}, 1 \leqq p \leqq 2$. Hence, with the usual terminology, $g(u)$ has a Fourier transform $G(x)$ in $L^{p^{\prime}}$. Further, by Plancherel's argument, $\dagger$ the relation (2.21) holds, the convergence being bounded in every interval in which $g(-u)$ is bounded. The desired conclusion therefore follows by the preceding argument.

The following lemma together with Lemma 3 completes the proof of Theorem 1.

LEMma 4. If $f(x)$ belongs to $H^{p}, 1 \leqq p \leqq \infty$, and if $F(x)$ is defined as in Lemma 3, then

almost everywhere.

$$
f(x)=(2 \pi)^{-1 / 2} \int_{-\infty}^{\infty} F(u) e^{i x u} d u
$$

In Theorems 3 and 4 take

Then

$$
g(u)= \begin{cases}1, & -x \leqq u \leqq 0 \\ 0, & u<-x, 0<u\end{cases}
$$

$$
G(y)=(2 \pi)^{-1 / 2} \int_{-x}^{0} e^{-i y u} d u=\left(\frac{2}{\pi}\right)^{1 / 2} \frac{\sin \frac{1}{2} x y}{y} e^{i x y / 2},
$$

and the hypotheses of Theorems 3 and 4 are obviously satisfied.

Hence

† Hobson, 5, p. 750. 


$$
\int_{0}^{x} f(u) d u=\left(\frac{2}{\pi}\right)^{1 / 2} \int_{-\infty}^{\infty} \frac{\sin \frac{1}{2} x y}{y} e^{i x y / 2} F(y) d y .
$$

Consider first the case $p=1$. In this case $F(y)$ belongs to $L(-\infty, \infty)$ and the second member of (2.31) may be written in the form

$$
(2 \pi)^{-1 / 2} \int_{0}^{x} d u \int_{-\infty}^{\infty} e^{i u y} F(y) d y .
$$

The desired conclusion now follows at once.

Now suppose $p>1$. From (2.31) and Hölder's inequality,

$$
\begin{aligned}
\left|\int_{0}^{x} f(u) d u\right| & \leqq\left(\frac{2}{\pi}\right)^{1 / 2}\left[\int_{-\infty}^{\infty}\left|\frac{\sin \frac{1}{2} x y}{y}\right|^{p} d y\right]^{1 / p^{\prime}}\left[\int_{-\infty}^{\infty}|F(y)| p d p\right]^{1 / p} \\
& \leqq M x^{1 / p}
\end{aligned}
$$

Take

$$
G(u)=\left\{\begin{array}{cl}
(2 \pi)^{-1 / 2}\left(1-\frac{|u|}{\omega}\right) e^{i x u}, & -\omega \leqq u \leqq \omega, \\
0, & u \leqq-\omega, \omega \leqq u .
\end{array}\right.
$$

Then

$$
g(-y)=\frac{2}{\pi \omega} \frac{\sin ^{2} \frac{1}{2} \omega(x-y)}{(x-y)^{2}}
$$

and the hypotheses of Theorems 3 and 4 are again satisfied. Hence

$$
\begin{aligned}
(2 \pi)^{-1 / 2} \int_{-\omega}^{\omega}( & \left.1-\frac{|u|}{\omega}\right) F(u) e^{i x u} d u \\
& =\frac{2}{\pi \omega} \int_{-\infty}^{\infty} f(x+y) \frac{\sin ^{2} \frac{1}{2} x y}{y^{2}} d y
\end{aligned}
$$

But it is easy to see that, in virtue of (2.32), the integral in the second member of (2.33) exists as an improper Lebesgue integral. For, writing

we have

$$
f_{1}(y)=\int_{0}^{y} f(u) d u
$$

$$
\begin{aligned}
\int_{0}^{Y} f(x+y) \frac{\sin ^{2} \frac{1}{2} \omega y}{y^{2}} d y= & f_{1}(x+Y) \frac{\sin ^{2} \frac{1}{2} \omega Y}{Y^{2}}-\frac{1}{4} \omega^{2} f_{1}(x) \\
& -\int_{0}^{Y} f_{1}(x+y) \frac{d}{d y}\left(\frac{\sin ^{2} \frac{1}{2} \omega y}{y^{2}}\right) d y,
\end{aligned}
$$

and by (2.32) this obviously tends to a limit as $Y$ tends to infinity. 
Divide the integral in the second member of (2.33) into three terms, viz.,

$$
\frac{2}{\pi \omega}\left\{\int_{-\infty}^{-K}+\int_{-K}^{K}+\int_{K}^{\infty}\right\}=I_{1}+I_{2}+I_{3},
$$

where $K$ is a positive constant such that $-K \leqq x \leqq K$. Then

$$
\begin{aligned}
I_{3}= & -\frac{2}{\pi \omega} \frac{\sin ^{2} \frac{1}{2} \omega K}{K^{2}} f_{1}(x+K)+\frac{4}{\pi \omega} \int_{K}^{\infty} \frac{\sin ^{2} \frac{1}{2} \omega y}{y^{3}} f_{1}(x+y) d y \\
& -\frac{2}{\pi} \int_{K}^{\infty} \frac{\sin \omega \nu}{y^{2}} f_{1}(x+y) d y .
\end{aligned}
$$

Now, by (2.32),

$$
\left|f_{1}(x+y)\right| \leqq M|x+y|^{1 / p} \text {. }
$$

Therefore, if $K$ and $x$ are fixed,

$$
\lim _{\omega \rightarrow \infty} I_{3}=-\frac{2}{\pi} \lim _{\omega \rightarrow \infty} \int_{K}^{\infty} \frac{\sin \omega y}{y^{2}} f_{1}(x+y) d y=0
$$

by the Riemann-Lebesgue theorem. Similarly $\lim _{\omega \rightarrow \infty} I_{1}=0$ while $I_{2}$ is Fejér's integral and so tends to $f(x)$ as $\omega$ tends to infinity. This yields the desired result.

3. The class $L_{p}^{*}$. The fundamental property of functions of this class is given in the following theorem. The corresponding result for the case $p=\infty$ is given elsewhere. $\dagger$

TheOREM 5. If $F(x)$ belongs to $L_{p}^{*}, 1 \leqq p<\infty$, and if

$$
f(x)=(2 \pi)^{-1 / 2} \frac{d}{d x} \int_{-\infty}^{\infty} \frac{e^{i x u}-1}{i u} F(u) d u,
$$

then $f(x)$ belongs to $H^{p}$ and $F(x)$ is the Fourier transform in $L^{p}$ of $f(x)$.

We begin by showing that $f(x)$ belongs to $H^{p}$. Since $F(x)$ belongs to $L_{p}^{*}$, $1 \leqq p<\infty$, the integral

$$
\int_{-\infty}^{\infty} \frac{e^{i x u}-1}{i u} F(u) d u
$$

converges uniformly in every finite range. We have

$$
F(x, \omega)=(2 \pi)^{-1 / 2} \int_{-\omega}^{\omega}\left(1-\frac{|u|}{\omega}\right) f(u) e^{-i x u} d u
$$

† Offord, 7, Theorem 5. 


$$
\begin{aligned}
& =(2 \pi)^{-1 / 2} \int_{-\omega}^{\omega} \frac{d}{d u}\left\{\left(1-\frac{|u|}{\omega}\right) e^{-i x u}\right\} d u \int_{-\infty}^{\infty} \frac{e^{i u t}-1}{i t} F(t) d t \\
& =(2 \pi)^{-1 / 2} \int_{-\infty}^{\infty} F(t) d t \int_{-\omega}^{\omega} \frac{d}{d u}\left\{\left(1-\frac{|u|}{\omega}\right) e^{-i x u}\right\} \frac{e^{i t u}-1}{i t} d u \\
& =\frac{2}{\pi \omega} \int_{-\infty}^{\infty} \frac{\sin ^{2} \frac{1}{2} \omega t}{t^{2}} F(t+x) d t .
\end{aligned}
$$

Consider first the case $1<p<\infty$. By Hölder's inequality

$$
\begin{aligned}
|F(x, \omega)|^{p} & \leqq\left(\frac{2}{\pi \omega}\right)^{p}\left[\int_{-\infty}^{\infty}|F(t+x)|^{p}\left|\frac{\sin \frac{1}{2} \omega t}{t}\right|^{p} d t\right]\left[\int_{-\infty}^{\infty}\left|\frac{\sin \frac{1}{2} \omega t}{t}\right|^{p^{\prime}} d t\right]^{p / p^{\prime}} \\
& \leqq M \omega^{1-p} \int_{-\infty}^{\infty}|F(t+x)|^{p}\left|\frac{\sin \frac{1}{2} \omega t}{t}\right|^{p} d t
\end{aligned}
$$

Hence

$$
\begin{aligned}
\int_{-\infty}^{\infty} \mid F(x, \omega) /^{p} d x & \leqq M \omega^{1-p} \int_{-\infty}^{\infty}\left|\frac{\sin \frac{1}{2} \omega t}{t}\right|^{p} d t \int_{-\infty}^{\infty}|F(t+x)|^{p} d x \\
& \leqq M \int_{-\infty}^{\infty}\left|\frac{\sin \theta}{\theta}\right|^{p} d \theta \int_{-\infty}^{\infty}|F(\xi)|^{p} d \xi=M,
\end{aligned}
$$

the desired result. Now suppose $p=1$. Then

$$
\int_{-\infty}^{\infty}|F(x, \omega)| d x \leqq \frac{2}{\pi \omega} \int_{-\infty}^{\infty} \frac{\sin ^{2} \frac{1}{2} \omega t}{t^{2}} d t \int_{-\infty}^{\infty}|F(t+x)| d x \leqq M .
$$

We have further to show that $F(x, \omega)$ satisfies (1.31). Let $e$ be any measurable set in $(-\infty, \infty)$. Then

$$
\int_{e}|F(x, \omega)| d x \leqq \frac{2}{\pi \omega} \int_{-\infty}^{\infty} \frac{\sin ^{2} \frac{1}{2} \omega t}{t^{2}} d t \int_{e}|F(x+t)| d x .
$$

But since $F(x)$ belongs to $L(-\infty, \infty)$ it is possible to find a $\delta$ independently of $t$ such that, if $m(e) \leqq \delta$, then

$$
\int_{e}|F(x+t)| d t \leqq \epsilon .
$$

Hence $f(x)$ belongs to $H^{1}$ as desired. It only remains to show that $F(x, \omega)$ tends to $F(x)$ almost everywhere. This, however, follows at once from the last equality in (3.11). This completes the proof of the theorem.

THEOREM 6. If $f(x)$ belongs to $H^{p}, 1 \leqq p \leqq \infty$, then its Fourier transform $F(x)$ belongs to $L_{p}^{*}$. 
The proof for the case $p=\infty$ is given elsewhere. $†$ When $1 \leqq p<\infty$, we have

$$
\begin{aligned}
(2 \pi)^{-1 / 2} & \int_{-\infty}^{\infty} \frac{e^{i x u}-1}{i u} F(u) d u \\
& =(2 \pi)^{-1 / 2} \lim _{\omega \rightarrow \infty} \int_{-\omega}^{\omega}\left(1-\frac{|y|}{\omega}\right) f(y) d y \int_{-\infty}^{\infty} \frac{\left(e^{i x u}-1\right) e^{-i y u}}{i u} d u \\
& =\int_{0}^{x} f(y) d y .
\end{aligned}
$$

This is the desired result.

The next theorem connects the theory developed here with the ordinary theory of Fourier transforms.

Theorem 7. Let $f(x)$ belong to $H^{p}, 1<p<\infty$, and let $F(x)$ be its Fourier transform in $L^{p}$ as defined in Theorem 1. Write

then

$$
I(x, \omega)=(2 \pi)^{-1 / 2} \int_{-\omega}^{\omega} f(u) e^{-i x u} d u
$$

$$
\lim _{\omega \rightarrow \infty} \int_{-\infty}^{\infty}|F(x)-I(x, \omega)|^{p} d x=0,
$$

i.e., $F(x)$ is the Fourier transform in $L^{p}$ of $f(x)$ in the ordinary sense. Conversely if $f(x)$ is such that (3.12) is satisfied, then $f(x)$ belongs to $H^{p}$ and $F(x)$ is its Fourier transform in $L^{p}$ in the sense of Definition 1.

To prove the first part of the theorem, in Theorems 3 and 4, take

Then

$$
g(u)=\left\{\begin{array}{cl}
(2 \pi)^{-1 / 2} e^{i x u}, & -\omega \leqq u \leqq \omega, \\
0, & u<-\omega, \omega<u .
\end{array}\right.
$$

Hence

$$
G(y)=\frac{1}{\pi} \frac{\sin \omega(x-y)}{x-y}
$$

$$
I(x, \omega)=(2 \pi)^{-1 / 2} \int_{-\omega}^{\omega} f(u) e^{-i x u} d u=\frac{1}{\pi} \int_{-\infty}^{\infty} F(y) \frac{\sin \omega(x-y)}{x-y} d y .
$$

Now Hille and Tamarkin $\ddagger$ have shown that the integral in the second member converges in mean of order $p, 1<p<\infty$, to $F(x)$ as $\omega$ tends to infinity. This proves the first part of the theorem. As regards the second part, we have

† Offord, 7, Theorem 2.

$\ddagger$ Hille and Tamarkin, 4, pp. 770-771. 
Hence, if $1<p<\infty$,

$$
F(t)=\underset{\omega \rightarrow \infty}{\lim } \int_{-\omega}^{\omega} f(u) e^{-i t u} d u .
$$

$$
\begin{aligned}
(2 \pi)^{-1 / 2} \int_{-\infty}^{\infty} \frac{e^{i x t}-1}{i t} F(t) d t & =(2 \pi)^{-1 / 2} \int_{-\infty}^{\infty} f(u) d u \int_{-\infty}^{\infty} \frac{e^{i x t}-1}{i t} e^{-i t u} d t \\
& =\int_{0}^{x} f(u) d u .
\end{aligned}
$$

Therefore $F(t)$ belongs to $L_{p}^{*}$ and so, by Theorem $5, f(x)$ must belong to $H^{p}$. Again, by Theorem 5, it follows that $F(t)$ is the Fourier transform in $L^{p}$ of $f(x)$. This is the desired result.

We now give some sufficient conditions for functions to belong to the classes $H^{p}$ and $L_{p}^{*}$.

THEOREM 8. If $F(x)$ belongs to $L^{p}(-\infty, \infty), 1 \leqq p \leqq \infty$, and if

$$
(2 \pi)^{-1 / 2} \int_{-\infty}^{\infty} F(u) e^{i x u} d u
$$

converges everywhere to a function $f(x)$ which is everywhere finite and integrable $L$ in every finite range, then $F(x)$ belongs to $L_{p}^{*}$ and is the Fourier transform in $L^{p}$ of $f(x)$.

Since $F(x)$ belongs to $L^{p}(-\infty, \infty), 1 \leqq p \leqq \infty$,

$$
\int_{-\infty}^{\infty} \frac{|F(x)|}{1+x^{2}} d x
$$

is finite. Now Pollard $\dagger$ has shown that if (3.2) converges everywhere to a function $f(x)$ which is everywhere finite and integrable $L$ in every finite range and if (3.3) is finite, then

$$
\begin{aligned}
\int_{0}^{x} d t \int_{0}^{t} f(u) d u= & (2 \pi)^{-1 / 2} \int_{-1}^{1} \frac{e^{i x u}-1-i x u}{-u^{2}} F(u) d u \\
& -(2 \pi)^{-1 / 2}\left\{\int_{1}^{\infty}+\int_{-\infty}^{-1}\right\} \frac{e^{i x u}}{u^{2}} F(u) d u+\text { constant }
\end{aligned}
$$

Hence, since $F(x)$ belongs to $L^{p}(-\infty, \infty), 1 \leqq p \leqq \infty$,

$$
f(x)=(2 \pi)^{-1 / 2} \frac{d}{d x} \int_{-\infty}^{\infty} \frac{e^{i x u}-1}{i u} F(u) d u,
$$

which is the desired result.

† Pollard, 11, p. 455. 
The following theorem also follows from Pollard's methods.

THEOREM 9. If $f(x)$ is such that

$$
\int_{-\infty}^{\infty} \frac{|f(x)|}{1+x^{2}} d x
$$

is convergent and if

$$
(2 \pi)^{-1 / 2} \int_{-\infty}^{\infty} f(u) e^{-i x u} d u
$$

converges everywhere to a function $F(x)$ which is everywhere finite and belongs to $L^{p}(-\infty, \infty), 1 \leqq p<\infty$, then $f(x)$ belongs to $H^{p}$ and $F(x)$ is its Fourier transform.

Without loss of generality we may suppose $f(u)=0,0 \leqq u \leqq 1$. Then it follows by Pollard's theorem that

$$
\frac{f(u)}{u^{2}}=-(2 \pi)^{-1 / 2} \lim _{Y \rightarrow \infty} \int_{-Y}^{Y}\left(1-\frac{|y|}{Y}\right) F_{2}(y) e^{i u y} d y
$$

where

$$
F_{2}(y)=\int_{0}^{v} F_{1}(t) d t=\int_{0}^{\nu} d t \int_{0}^{t} F(u) d u .
$$

Now Pollard has shown that

$$
F_{2}(y) \rightarrow 0
$$

as $y$ tends to infinity. Again, since $F(y)$ belongs to $L^{p}, 1 \leqq p<\infty$,

$$
\left|F_{1}(y)\right| \leqq y^{1 / p^{\prime}}\left[\int_{-\infty}^{\infty}|F(t)| p d t\right]^{1 / p}=o(y) .
$$

Hence, from (3.41), (3.42), (3.43) and (3.44),

$$
\begin{aligned}
\int_{0}^{x} f(u) d u & =\int_{1}^{x} f(u) d u=-(2 \pi)^{-1 / 2} \lim _{Y \rightarrow \infty} \int_{-Y}^{Y}\left(1-\frac{|y|}{Y}\right) F_{2}(y) d y \int_{1}^{x} u^{2} e^{i y u} d u \\
& =(2 \pi)^{-1 / 2} \lim _{Y \rightarrow \infty} \int_{-Y}^{Y}\left(1-\frac{|y|}{Y}\right) F_{2}(y) \frac{d^{2}}{d y^{2}}\left(\frac{e^{i x y}-e^{i y}}{i y}\right) d y \\
& =(2 \pi)^{-1 / 2} \lim _{Y \rightarrow \infty} \int_{-Y}^{Y} \frac{e^{i x y}-e^{i y}}{i y} \frac{d^{2}}{d y^{2}}\left\{\left(1-\frac{|y|}{Y}\right) F_{2}(y)\right\} d y \\
& =(2 \pi)^{-1 / 2} \int_{-\infty}^{\infty} \frac{e^{i x y}-1}{i y} F(y) d y-(2 \pi)^{-1 / 2} \int_{-\infty}^{\infty} \frac{e^{i y}-1}{i y} F(y) d y,
\end{aligned}
$$


the integrals being convergent. It follows that $F(x)$ belongs to $L_{p}^{*}$ and conquently, by Theorem $5, f(x)$ belongs to $H^{p}$. This is the desired result.

4. The class $H^{p} L^{q}$. Consider the class $H^{p} L^{q}$ of all the functions which belong to both $H^{p}, 1 \leqq p \leqq \infty$, and $L^{q}, 1 \leqq q \leqq \infty$. The following theorem holds for functions of this class.

THEOREM 10. If $f(x)$ belongs to $H^{p} L^{q}, 1 \leqq p \leqq \infty, 1 \leqq q \leqq \infty$, then it has a Fourier transform $F(x)$ in $L^{p} . F(x)$ belongs to $H^{q} L^{p}$ and $f(-x)$ is the Fourier transform in $L^{a}$ of $F(x)$.

Consider first the case $1 \leqq p<\infty, 1 \leqq q \leqq \infty$. We can apply Theorems 3 and 4 with

Then

$$
G(u)= \begin{cases}1, & 0 \leqq u \leqq x \\ 0, & \text { elsewhere }\end{cases}
$$

$$
\int_{0}^{x} F(u) d u=(2 \pi)^{-1 / 2} \int_{-\infty}^{\infty} \frac{1-e^{-i x u}}{i u} f(u) d u .
$$

But $f(x)$ belongs to $L^{q}$ and so by (4.1) to $L_{q}^{*}$. Hence, if $1 \leqq q<\infty$, it follows from Theorem 5 that $F(x)$ belongs to $H^{q}$. Again, if $q=\infty$ then $F(x)$ belongs to $H^{\infty}$ by the analogue $\dagger$ of Theorem 5 for this case. Now $f(x)$ belongs to $H^{p}$ and so must have a Fourier transform in $L^{p}$, i.e., $F(x)$ belongs to $H^{q} L^{p}$. Also, since $F(x)$ belongs to $H^{q}$, it has a Fourier transform $f(-x)$ in $L^{q}$. This proves the theorem for the case $1 \leqq p<\infty$. Now suppose $p=\infty$. Then

$$
F(x)=(2 \pi)^{-1 / 2} \lim _{\omega \rightarrow \infty} \int_{-\omega}^{\omega}\left(1-\frac{|u|}{\omega}\right) f(u) e^{-i x u} d u .
$$

The convergence is bounded so that

$$
\int_{0}^{x} F(t) d t=(2 \pi)^{-1 / 2} \lim _{\omega \rightarrow \infty} \int_{-\omega}^{\omega}\left(1-\frac{|u|}{\omega}\right) \frac{1-e^{-i x u}}{i u} f(u) d u .
$$

The desired result now follows as before.

From this result we can deduce at once the following theorem.

THEOREM 11. If $f(x)$ has a Fourier transform $F(x)$ in the class $L^{p}, 1 \leqq p \leqq \infty$, then the necessary and sufficient condition that $f(-x)$ should be the Fourier transform of $F(x)$ in $L^{q}, 1 \leqq q \leqq \infty$, is that $f(x)$ should belong to $L^{q}$.

It must be remarked that this theorem is true only when the Fourier transform of a function is defined as in Definition 1.

† Offord, 7, p. 208. 
5. The reciprocal class $H^{p} L^{p}$. A very interesting special case of the class $B^{p} L^{q}$ is the class $H^{p} L^{p}$ of all the functions which belong to both $H^{p}$ and $L^{p}$. We obtain the following theorem by putting $q=p$ in Theorem 10 .

THEOREM 12. If $f(x)$ belongs to $H^{p} L^{p}, 1 \leqq p \leqq \infty$, then its Fourier transform also belongs to $H^{p} L^{p}$.

We have already discussed $\dagger$ the case $p=\infty$ under name of the class $H B$. We now add an example of a function which is bounded and belongs to $H^{\infty}$ but does not belong to any Lebesgue class. Take

$$
f(u)=\cos \left\{\left[\frac{u}{2 \pi}\right] u\right\}=\left\{\begin{array}{cl}
1, & 0 \leqq u<2 \pi, \\
\cos n u, & 2 n \pi \leqq u<2(n+1) \pi .
\end{array}\right.
$$

We shall show that

$$
\left|\int_{0}^{\infty} f(u) \cos x u d u\right| \leqq M
$$

for all $x$ and $\omega$ and from this it follows that

$$
\left|\int_{-\omega}^{\omega}\left(1-\frac{|u|}{\omega}\right) e^{-i x u} f(u) d u\right| \leqq M,
$$

i.e., $f(u)$ belongs to $H^{\infty} L^{\infty}$ as desired. Suppose that $2(m+1) \pi \leqq \omega<2(m+2) \pi$ and that $r-\frac{1}{2} \leqq x<r+\frac{1}{2}$. Then, using $O(1)$ to denote an expression which is uniformly bounded in $x$ and $\omega$, we get

$$
\begin{aligned}
& \int_{0}^{\infty} f(u) \cos x u d u=\sum_{\substack{n=0 \\
n \neq r-1, r}}^{m} \int_{2 n}^{2(n+1) \pi} \cos x u \cos n u d u+O(1) \\
&= \frac{1}{2} \sum_{\substack{n=0 \\
n \neq r-1, r}}^{m}\left[\frac{\sin 2(n+1) \pi x-\sin 2 n \pi x}{n+x}\right. \\
&=\frac{1}{2} \sum_{\substack{n=1 \\
n \neq r-1, r, r+1}}^{m} \sin 2 n \pi x\left\{\frac{\sin 2(n+1) \pi x-\sin 2 n \pi x}{(n+x)(n+x-1)}\right]+O(1) \\
&-
\end{aligned}
$$

Therefore

† Offord, 7, p. 211. 


$$
\begin{aligned}
&\left|\int_{0}^{\infty} f(u) \cos x u d u\right| \\
& \leqq \frac{1}{2} \sum_{\substack{n=1 \\
n \times r-1, r, r+1}}^{m}\left\{\frac{1}{(n+x)(n+x-1)}+\frac{1}{|n+x||n-x-1|}\right\}+O(1) \\
& \leqq \frac{1}{2} \sum_{n=1}^{\infty} \frac{1}{n^{2}}+\frac{1}{2} \sum_{n=r+2}^{\infty} \frac{1}{(n-x)(n-x-1)} \\
&+\frac{1}{2} \sum_{n=1}^{r-2} \frac{1}{(x-n)(x-n+1)}+O(1) \\
& \leqq \frac{3}{2} \sum_{n=1}^{\infty} \frac{1}{n^{2}}+O(1) \leqq M .
\end{aligned}
$$

It is easily verified that

$$
\begin{aligned}
F(x) & =(2 \pi)^{-1 / 2} \int_{0}^{\infty} \cos x u \cos \left\{\left[\frac{u}{2 \pi}\right] u\right\} d u \\
& =(2 \pi)^{-1 / 2} 2 x \sin \pi x \sum_{n=0}^{\infty} \frac{\cos (2 n+1) \pi x}{x^{2}-n^{2}} .
\end{aligned}
$$

Corresponding to each class $B^{p} L^{p}$ there is a class of self-reciprocal functions and we conclude this paper by giving two theorems for these functions.

Suppose that $\chi(t)$ is integrable $L$ in every finite range and such that

$$
\int_{-\infty}^{\infty}\left|\int_{-T}^{T}\left(1-\frac{|t|}{T}\right) \chi(t) x^{-1 / 2-i t} d t\right|^{p} d x \leqq M
$$

for all $T \geqq 0$. Further let

$$
\frac{\chi(t)}{2^{1 / 4+i t / 2} \Gamma\left(\frac{1}{4}+\frac{1}{2} i t\right)}=\text { even function of } t .
$$

THEOREM 13. A necessary and sufficient condition that an even function $f(x)$ of $H^{p} L^{p}, 1 \leqq p \leqq \infty$, should be its own Fourier transform is that it should be of the form

$$
f(x)=\frac{1}{2 \pi} \int_{-\infty}^{\infty} \chi(t) x^{-1 / 2-i t} d t,
$$

where the integral is summable $(C, 1)$ almost everywhere and $\chi(t)$ satisfies $(5.1)$ and (5.2). 
THEOREM 14. A necessary and sufficient condition that an even function $f(x)$ of $L^{p}, 1 \leqq p<\infty$, should be a solution of the equation

$$
f(x)=(2 \pi)^{-1 / 2} \frac{d}{d x} \int_{-\infty}^{\infty} \frac{e^{i x u}-1}{i u} f(u) d u
$$

is that it should be of the form (5.3), where the integral is summable $(C, 1)$ almost everywhere and $\chi(t)$ satisfies (5.1) and (5.2).

These theorems are the analogues for the class $H^{p} L^{p}$ of the theorem of Hardy and Titchmarsh $\dagger$ for the class $L^{2}$. They can be proved by their argument and we shall not give the proof here. It is interesting to notice that the function $x^{1 / 2} J_{-1 / 4}\left(\frac{1}{2} x^{2}\right)$ is a self-reciprocal function of the class $H^{p} L^{p}$, for all $p>2$, but it does not belong to $L^{2}$.

\section{REFERENCES}

1. S. Banach, Théorie des Opérations Linéaires, 1932.

2. A. C. Berry, The Fourier transform identity theorem, Annals of Mathematics, (2), vol. 32 (1931), pp. 227-232.

3. G. H. Hardy and E. C. Titchmarsh, Self-reciprocal functions, Quarterly Journal (Oxford Series), vol. 1 (1930), pp. 196-231.

4. E. Hille and J. D. Tamarkin, On the theory of Fourier transforms, Bulletin of the American Mathematical Society, vol. 39 (1933), pp. 768-774.

5. E. W. Hobson, Functions of a Real Variable, 2d edition, vol. 2, 1926.

6. H. Lebesgue, Sur les intégrales singulières, Annales de Toulouse, (3), vol. 1 (1909), pp. 25-128.

7. A. C. Offord, On Fourier transforms, Proceedings of the London Mathematical Society, (2), vol. 38 (1934), pp. 197-216.

8. A. C. Offord, On Hankel transforms, Proceedings of the London Mathematical Society, (2), vol. 39 (1935), pp. 49-67.

9. A. C. Offord, Fourier and Hankel transforms, British Association Report, 1933, pp. 455-456.

10. A. C. Offord, On Fourier transforms (II), Proceedings of the London Mathematical Society, (2). In press.

11. S. Pollard, Identification of the coefficients in a trigonometrical integral, Proceedings of the London Mathematical Society, (2), vol. 25 (1926), pp. 451-468.

12. N. Wiener, The Fourier Integral, Cambridge, 1933.

$\dagger$ Hardy and Titchmarsh, 3, p. 201. See also Offord, 7, Theorem 9.

St. John's College, CAMBRIDGe, ENGLAND 